\title{
Letter
}

\section{Three-Dimensional Nanoscopy of Colloidal Crystals}

\author{
Benjamin Harke, Chaitanya K. Ullal, Jan Keller, and Stefan W. Hell
}

Nano Lett., 2008, 8 (5), 1309-1313• DOI: 10.1021/nl073164n • Publication Date (Web): 01 January 2008

Downloaded from http://pubs.acs.org on March 23, 2009
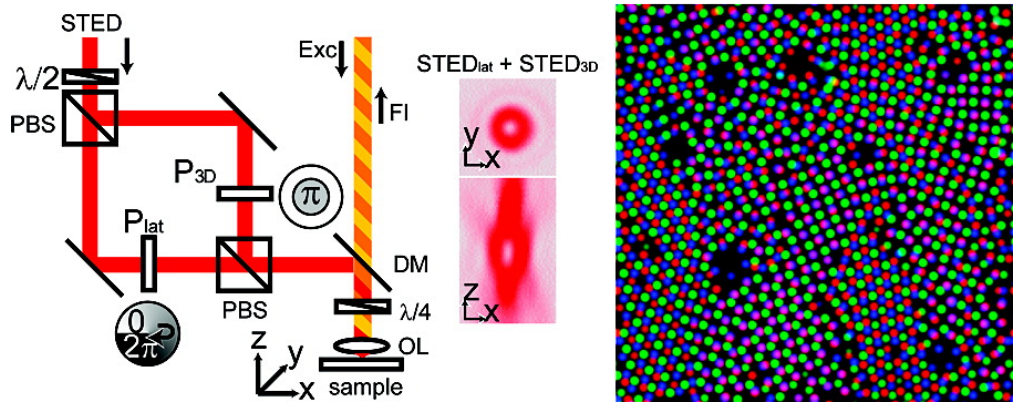

\section{More About This Article}

Additional resources and features associated with this article are available within the HTML version:

- $\quad$ Supporting Information

- Links to the 3 articles that cite this article, as of the time of this article download

- $\quad$ Access to high resolution figures

- Links to articles and content related to this article

- Copyright permission to reproduce figures and/or text from this article

\section{View the Full Text HTML}




\title{
Three-Dimensional Nanoscopy of Colloidal Crystals
}

\author{
Benjamin Harke, ${ }^{\dagger}$ Chaitanya K. Ullal, ${ }^{, \dagger}$ Jan Keller, and Stefan W. Hell ${ }^{\star}$ \\ Department of Nanobiophotonics, Max Planck Institute for Biophysical Chemistry, \\ Am Fassberg 11, 37077 Göttingen, Germany
}

Received December 4, 2007

\begin{abstract}
We demonstrate the direct three-dimensional imaging of densely packed colloidal nanostructures using stimulated emission depletion microscopy. A combination of two de-excitation patterns yields a resolution of $43 \mathrm{~nm}$ in the lateral and $125 \mathrm{~nm}$ in the axial direction and an effective focal volume that is by 126 -fold smaller than that of a corresponding confocal microscope. The mapping of a model system of spheres organized by confined convective assembly unambiguously identified face-centered cubic, hexagonal close-packed, random hexagonal close-packed, and body-centered cubic structures.
\end{abstract}

An increasing need for noninvasive visualization on the nanoscale has fueled the development of far-field optical microscopy with resolution far below the wavelength of light. ${ }^{1,2}$ In materials science, structural studies with length scales of interest in the (sub-) micrometer range have typically been conducted either by collective scattering-based techniques or electron and scanning probe microscopes. Farfield optical methods however retain the advantage of simultaneously providing local, dynamic, and noninvasive three-dimensional (3D) structural information. Confocal fluorescence microscopy has thus emerged as a popular tool for the study of colloids as models for atomic and molecular systems. ${ }^{3-8}$ The diffraction-limited resolution of confocal microscopy however restricts the minimum size of the colloidal particles that can be employed. While in the case of ideal hard spheres the size of the sphere is not a control parameter, it affects sedimentation and their overall dynamics, requiring in many cases both density and refractive index matching. As a result, the variety of systems that can be studied is severely restricted.

Importantly, there has been considerable interest in colloids with more complex interactions and shapes. ${ }^{9-11}$ Allowing such systems to self-assemble results in a rich diversity of structures, ${ }^{12,13}$ which at the nanoscale can exhibit enhanced properties that are due to both their crystal structure and the constituent particles size. ${ }^{14}$ The size and orientation of the particles also affect the relative magnitudes of the attractive and repulsive forces that govern the process of self-assembly. Effects specific to the nanoscale regime, where the width of

* To whom correspondence should be addressed. E-mail: (SWH) hell@4pi.de; (CKU) ckullal@alum.mit.edu. Phone: +49 551201 2500. Fax: +495512012505.

Contributed equally to the work. the screening layer is commensurate with the size of the colloidal particles have been observed, such as the formation of nonclose-packed diamondlike lattices. ${ }^{15}$ Unfortunately, the unambiguous identification of 3D morphologies at length scales below the wavelength of light is challenging and/or tedious with established techniques. Here, we demonstrate the power of emerging far-field fluorescence microscopy with nanoscale resolution for the study of colloidal self-assembly. By simultaneously improving both the lateral $(x, y)$ and the axial $(z)$ resolution of stimulated emission depletion (STED) microscopy, we first report a general improvement in 3D resolution in far-field fluorescence microscopy and then apply the improved 3D resolution to uniquely access the stacking sequence of 3D crystals of nanoscale colloidal particles.

STED microscopy is an implementation of a family of scanning far-field optical techniques that exploit reversible saturable/switchable (fluorescence) transitions to achieve subdiffraction resolution. ${ }^{1,16,17}$ The STED method typically involves the interrogation of fluorescently labeled samples by a focused excitation beam overlaid with a red-shifted deexcitation or "STED beam". The de-excitation beam is reshaped in such a manner that when focused has a spatially varying intensity profile featuring an isolated intensity zero steeply bordered by bright de-excitation light peaks. The deexcitation light quenches excited molecules via stimulated emission. Increasing the power of the de-excitation light raises the effectiveness of the quenching ${ }^{18}$ thereby confining effective excitation and hence fluorescence emission to increasingly smaller areas around the isolated intensity zero. The confinement can be effected in all directions using suitable de-excitation patterns. Scanning the reduced fluorescence spot through the specimen yields subdiffraction 3D resolution. 

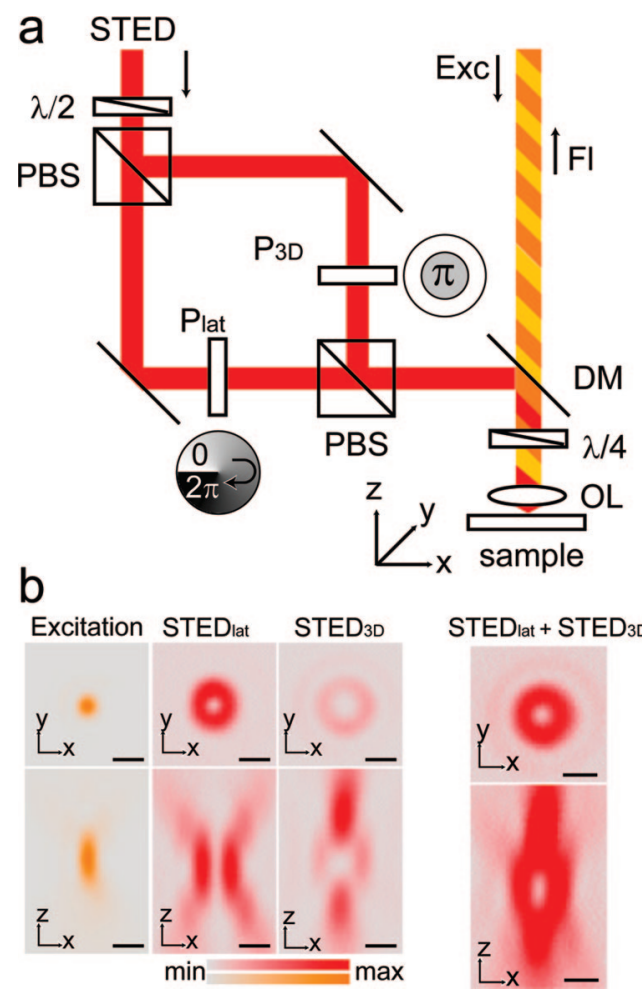

Figure 1. 3D STED microscopy for simultaneously increasing the resolution in the focal plane and along the optic axis. (a) Schematic setup. The STED power is distributed between the two phase plates $\left(\mathrm{P}_{\text {lat }}\right.$ and $\left.\mathrm{P}_{3 \mathrm{D}}\right)$ by using a combination of a $\lambda / 2$ plate and a polarizing beam splitter (PBS). The second PBS recombines the two beams incoherently. The excitation (Exc) and STED beams are overlaid by a dichroic mirror (DM). A $\lambda / 4$ plate ensures the circular polarization of all beams prior to being focused by the objective lens (OL). The fluorescence signal (Fl) is collected by the same lens. (b) Focal intensity distributions of excitation and STED beams measured using gold beads in reflectance mode. From left to right: Excitation, STED beam from $\mathrm{P}_{\text {lat }}$ arm resulting in the focal deexcitation pattern $S_{T E D}$ lat STED beam from $\mathrm{P}_{3 \mathrm{D}}$ arm yielding STED $_{3 \mathrm{D}}$, incoherent combination of both arms $\left(30 \% \mathrm{STED}_{\text {lat }} / 70 \%\right.$ $\mathrm{STED}_{3 \mathrm{D}}$ power distribution). The latter distribution results in an efficient coverage of the volume around the focal point. Scale bars $500 \mathrm{~nm}$.

The de-excitation patterns are created by inserting an appropriate phase shifting mask in the path of the STED beam, thereby reshaping the de-excitation beam in the focal area. A systematic comparison of different phase masks for use in STED microscopy identified two distinct phase masks as the optimum choice for confining the fluorescence spot in the lateral and axial directions, respectively. ${ }^{19}$ The use of a circular $2 \pi$ ramp phase mask $\left(\mathrm{P}_{\text {lat }}\right)$ was shown to yield an effective increase in focal plane (lateral) resolution. However, while it gives a steep de-excitation pattern in the focal plane, $\mathrm{P}_{\text {lat }}$ does not feature any de-excitation intensity on the optic axis, ${ }^{20}$ leaving the axial resolution unaffected. On the other hand, a phase mask consisting of a central circular area with a $\pi$-phase retardation $\left(\mathrm{P}_{3 \mathrm{D}}\right)$ was identified as the best de-excitation pattern for confinement in the axial direction. ${ }^{21}$ In both cases, the full width half-maximum (FWHM) of the fluorescent spot, which is tantamount to the
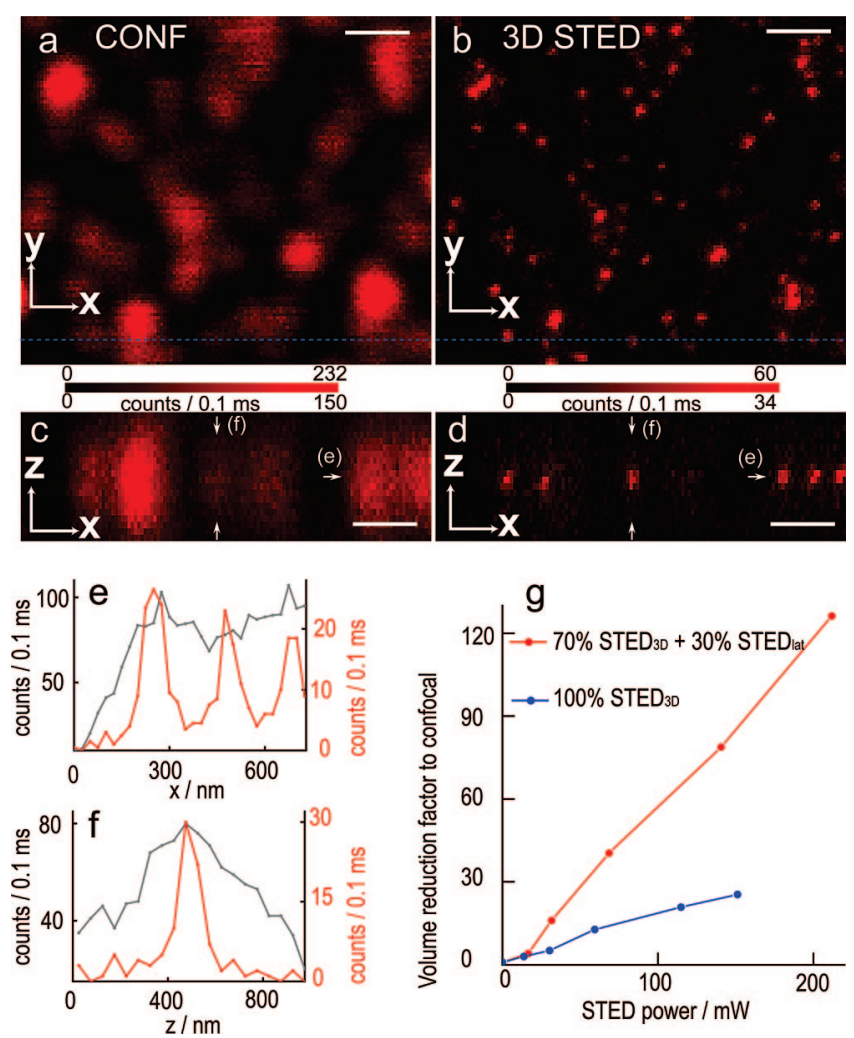

Figure 2. $(a-d) 3 D$ nanoscale image of dilute distribution of 20 nm diameter fluorescent spheres on glass. $x y$ sections of (a) confocal and (b) STED. (c) Confocal and (d) STED $x z$ sections along the dashed blue line indicated in panels a and $\mathrm{b}$. Individual beads can be easily resolved in the STED images. Comparing panel $\mathrm{c}$ with panel $\mathrm{d}$, note the significant reduction in cross-sectional area in the STED $x z$-image (e,f) Intensity profile along the (e) $x$ and (f) $z$ direction for sections indicated by the white arrows in panels $\mathrm{c}$ and d. All presented data is raw data. (g) Focal volume reduction relative to confocal focal volume measured using $20 \mathrm{~nm}$ fluorescent spheres. The combination of two de-excitation patterns gives a maximal volume reduction factor of 125 . Scale bars $1 \mu \mathrm{m}$.

effective point-spread function (PSF) of the system, is given by

$$
d_{i} \approx D_{i} / \sqrt{1+a_{i} I / I_{\mathrm{S}}}
$$

where $i=x, y, z$, and $D_{i}$ denotes the corresponding FWHM of the main diffraction spot in the particular direction, and $0.3<a_{i}<1$ takes into account the intensity profile of the depletion beam. $I$ is the intensity at the peaks of the deexcitation light, and $I_{\mathrm{S}}$ is a dye-characteristic intensity, which can be physically interpreted as the point at which the stimulated emission process dominates over other competing processes. The value is frequently assigned to the intensity at which half of the excited molecules are quenched by the applied STED beam. For $\lambda \approx 750 \mathrm{~nm}$ and focal doughnut areas of $10^{-9} \mathrm{~cm}^{2}, I_{\mathrm{S}}$ typically corresponds to $\approx 2 \mathrm{MW} / \mathrm{cm}^{2}$.

In the common situation of a sample without a preferential orientation, the dimension with the poorest resolution is the most limiting one. The 3D imaging of colloidal crystals thus requires a uniform resolution increase in all directions. Once a critical resolution in all dimensions is achieved, small focal volumes are desirable. This allows for the acquisition of the largest amount of spatial frequency information. Thus, although it is not a rigorous measure, for nearly spherical 


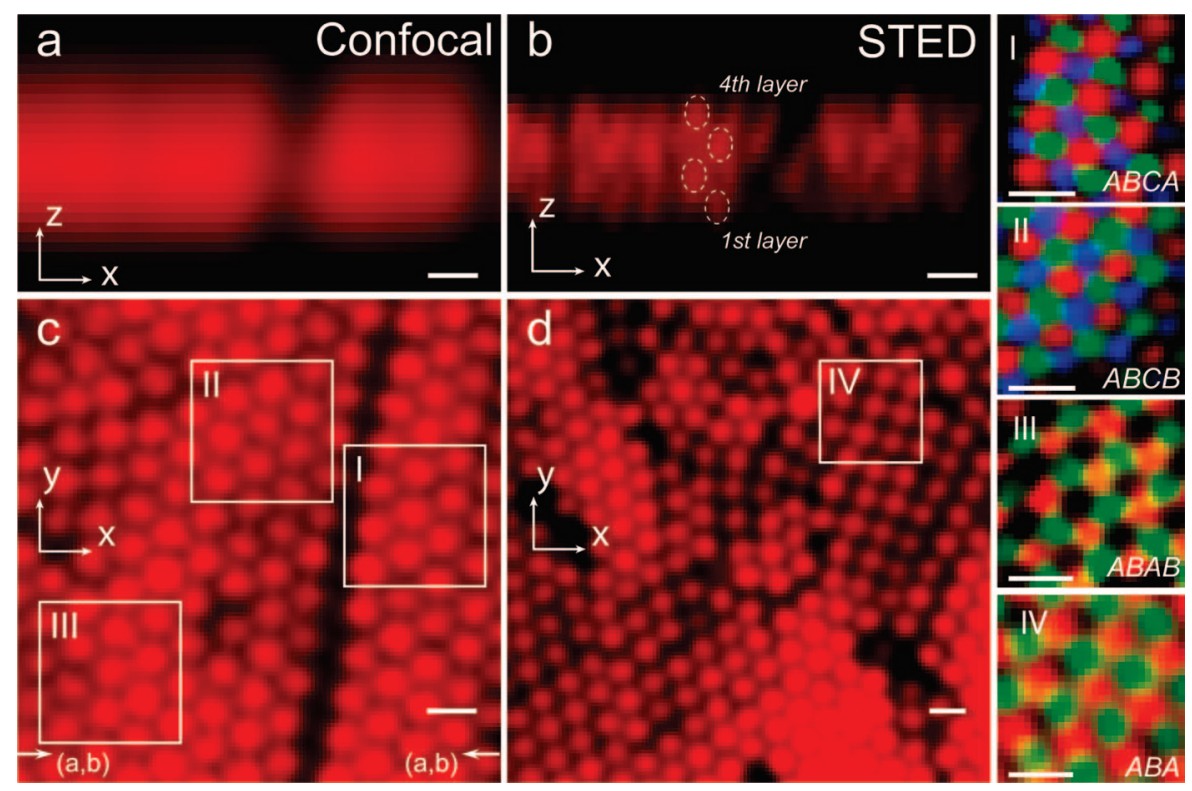

Figure 3. 3D nanoscopy of convectively assembled 3D colloidal crystals. (a,b) $x z$ section of a 3D data stack from (a) confocal and (b) STED image of a four layer crystal. (c) $x y$ section of the first layer of the four layer crystal shown in panels a and b. (d) $x y$ section of the first layer of a 3 layer crystal showing square packing as well hexagonal close packing. (e) Projection images of insets I (fcc, ABCA sequence), II (r-hcp, ABCB sequence), III (hcp, ABA sequence), and IV (bcc, ABA sequence). All data in this figure was linearly deconvolved. Scale bars $250 \mathrm{~nm}$.

spots the "focal volume", as defined by the FWHM of the effective PSF, can be used to assess the 3D-resolving power of the system. We estimate this focal volume, $V$, by

$$
V=\prod_{i} d_{i}
$$

In the regime where $I / I_{S} \gg 1$ we have

$$
V_{\mathrm{STED}} \propto \frac{V_{\text {confocal }}}{\left(I / I_{\mathrm{S}}\right)^{3 / 2}}
$$

In most practical applications, $I$ is limited by the onset of photobleaching thereby necessitating the identification of optimal de-excitation patterns. To target a high resolution in 3D or small focal volumes, we simultaneously employed both phase modifications $\mathrm{P}_{\text {lat }}$ and $\mathrm{P}_{3 \mathrm{D}}$ of the STED beams, as sketched in Figure 1. Incoherent superposition of the light fields of the resultant focal de-excitation patterns (PSFs), herein referred to as $\mathrm{STED}_{\text {lat }}$ and $\mathrm{STED}_{3 \mathrm{D}}$, respectively, allowed us to balance the strength of their specific confinement by tuning their relative power distribution through the combined use of a half-wave plate and a polarizing beam splitter. The incoherent superposition of STED lat and STED $3 \mathrm{D}$ was facilitated by the fact that our STED microscope was operated in a pulsed mode. The STED pulses were delivered by a mode-locked Ti:Sapphire laser emitting $<100$ fs pulses at $\lambda=750 \mathrm{~nm}$. To optimize STED, the pulses were stretched to a duration of $200 \mathrm{ps}$. Incoherence between $\mathrm{STED}_{\text {lat }}$ and $\mathrm{STED}_{3 \mathrm{D}}$ is simply ensured by applying a temporal delay slightly greater than $250 \mathrm{fs}$ between the respective pulse trains. Because this delay is much smaller than the $\sim 2 \mathrm{~ns}$ excited-state lifetime, the de-excitation efficiency of the two de-excitation focal patterns is not compromised.

The PSF of the excitation beam as well as STED lat and $\mathrm{STED}_{3 \mathrm{D}}$ were probed using a $150 \mathrm{~nm}$ diameter gold bead
(Figure 1b). A quarter wave plate ensured the circular polarization of all three beams. Because of the polarization dependence inherent in the method it was not possible to measure the combination of $\mathrm{STED}_{\text {lat }}$ and $\mathrm{STED}_{3 \mathrm{D}}$ directly. However, from the map of the individual PSF it is clear that an incoherent combination of the two STED beams would effectively cover the volume around the focal point.

Fluorescent polystyrene beads $(20 \mathrm{~nm}$, crimson beads, Invitrogen) were used to measure the focal volume as a function of the total power of $\mathrm{STED}_{\text {lat }}$ and $\mathrm{STED}_{3 \mathrm{D}}$. In a first configuration, $30 \%$ of the STED power was placed in $\mathrm{STED}_{\text {lat }}$ and the remaining part in $\mathrm{STED}_{3 \mathrm{D}}$, while in the second the distribution was reversed. Although simulations had suggested that the $70 \% \mathrm{STED}_{\text {lat }}$ plus $30 \% \mathrm{STED}_{3 \mathrm{D}}$ distribution would display the smallest focal volume, we found only slight variations between the two configurations. The $30 \% \mathrm{STED}_{\text {lat }}$ plus $70 \% \mathrm{STED}_{3 \mathrm{D}}$ combination was thus chosen because it concentrated more power in the confinement of fluorescence along the $z$-axis, the direction that displayed the lowest enhancement.

Figure $2 \mathrm{~g}$ shows a plot of the reduction in the focal volume as compared to a confocal microscope, along with that of the single de-excitation pattern created by $\mathrm{STED}_{3 \mathrm{D}}$. It should be noted that a slightly lower maximal power was applied in the case of the single pattern due to practical limitations in the setup, which was used for both single and combination measurements. The reduction in focal volume of the combination de-excitation pattern was consistently 2-3 times stronger than that of the single pattern but at the expense of an increased ellipticity of the focal spot. A maximal reduction from $162 \mathrm{aL}$ (confocal) to $1.3 \mathrm{aL}$ (combination de-excitation) at a maximal average power of $211 \mathrm{~mW}$ in the focal plane was obtained. This corresponds to a focal volume that is 126 

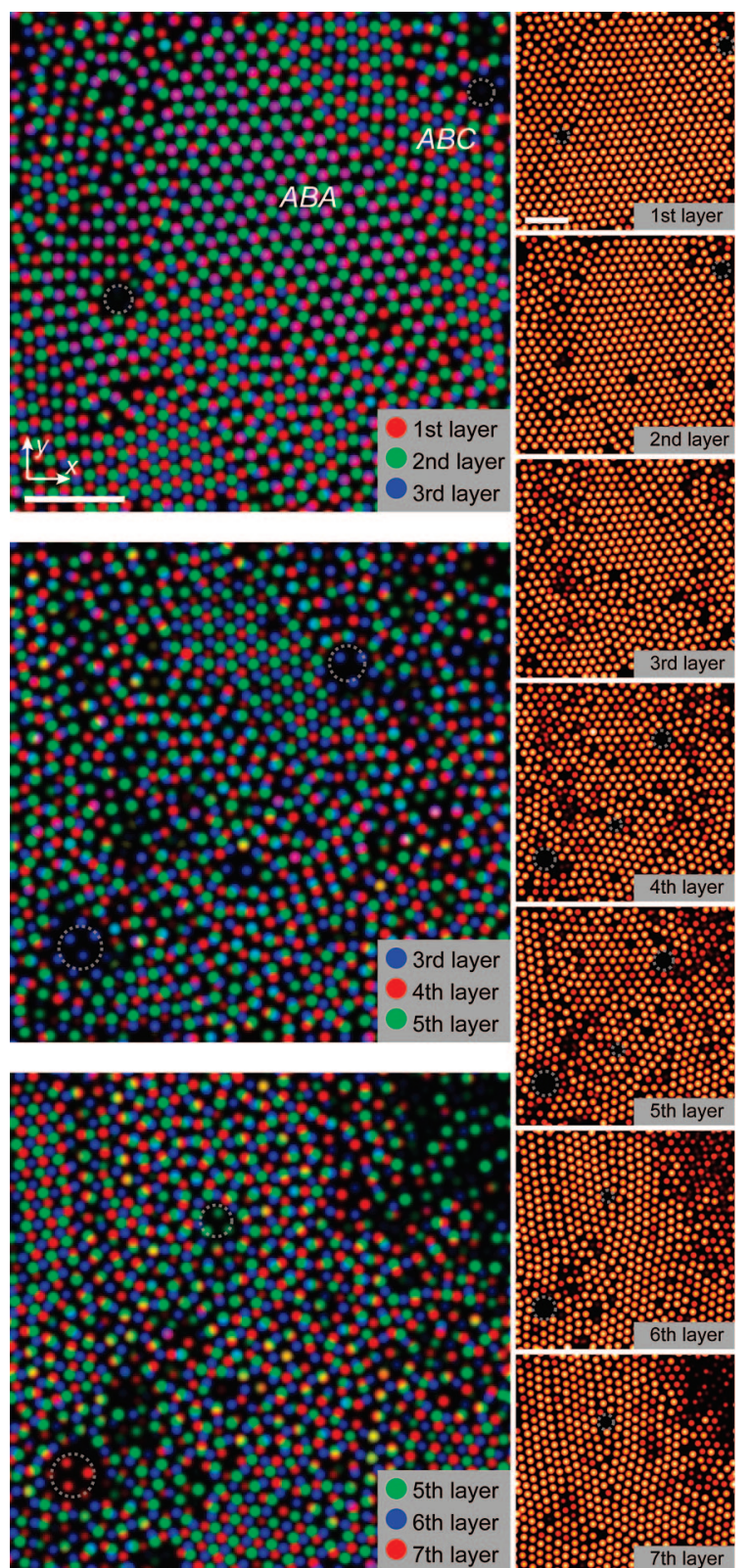

Figure 4. Image of localized spheres from a seven layer sample. Oversized spheres are replaced by circles with dotted gray outlines. The right-hand side column shows the individual layers. The column on the left shows a projection image of the layers taken three at a time. Spheres are colorized on the basis of the layers as indicated. The net packing is r-hcp. Both well-organized areas and the structural features around defects are clearly evident. Scale bar 1 micron.

times smaller than that of a corresponding confocal microscope and currently constitutes the smallest focal fluorescent volume suitable for 3D imaging produced with a single lens.

The 3D resolution was measured by imaging a dilute distribution of $20 \mathrm{~nm}$ polystyrene beads on a glass slide. Figure 2a,b shows the STED and for comparison confocal images in the $x y$ plane, while Figure 2c,d shows the $x z$ plane indicated by the blue lines in Figure 2a,b. These panels of Figure 2, along with the line profiles along the $z$ and $x$ directions, Figure 2e,f, respectively, clearly demonstrate the increased 3D resolution. Simultaneous resolutions of $43 \mathrm{~nm}$ in the lateral and $125 \mathrm{~nm}$ in the axial direction were obtained.
To demonstrate the ability of STED microscopy to unambiguously map the morphology of 3D colloidal crystal nanostructures, a model system was examined consisting of convectively assembled fluorescent latex spheres of $200 \mathrm{~nm}$ diameter. Convective assembly is a technique that involves the self-organization of spheres at the moving contact line created by the controlled evaporation of a solvent. ${ }^{22-24}$ While the exact details of the assembly process are not fully established, the flow of solvent compensating for the evaporation from the crystal surface, the volume fraction of particles, and the shape of the meniscus at the contact line are factors known to be involved in determining the resultant crystal structure. ${ }^{25,26}$ Under appropriate conditions the spheres form a hexagonally close-packed monolayer parallel to the substrate. The registration between subsequent close-packed layers determines whether the resultant crystal structure is face-centered cubic ( $\mathrm{ABCABC}$... sequence, fcc for short), hexagonal close packed (ABABA... sequence, hcp) or random hexagonal close- (r-hcp) packed. Using micron-sized hard spheres, it has been verified by optical microscopy that as the layer thickness and number of layers increase the structure alternates between being close-packed and bodycentered cubic (bcc). ${ }^{27}$

Because in the present examples we are dealing with hard spheres, similar behavior can also be expected at smaller scales that are accessible only by our method. Therefore, colloidal crystals of varying thicknesses were assembled by employing a variant of the convective assembly method known as confined convective assembly. ${ }^{28,29}$ The crystals were backfilled with a silicone refractive index oil so as to mitigate the effect of scattering and were imaged. Representative, 3D linearly deconvolved images of the resultant 3D structures examined are shown in Figure 3. The deconvolution was performed using a 3D Lorentzian function that was matched in both lateral and axial directions to the corresponding measured effective PSF. Figure $3 \mathrm{c}$ shows the first layer of a four layer crystal. The structure is close packed; an examination of the 3D structure shows regions of fcc (region I), r-hcp (region II), and hcp (region III).

Figure $3 \mathrm{~b}$ shows the STED image of the $x z$ section of the four layer crystal for the plane indicated by the arrows in Figure 3c. The corresponding confocal image is shown for comparison in Figure 3a. Figure 3d shows the first layer of a three layer crystal in a region that displays both fcc (region II) and bcc (region III) structures. The registration between the various layers can clearly be seen in the projection images shown in Figure 3e. The layer sequence for the regions I, II, III, and IV are $\mathrm{ABCA}, \mathrm{ABCB}, \mathrm{ABAB}$, and $\mathrm{ABA}$ respectively.

During the imaging process, no prior knowledge of the samples was exploited. In light of the fact that the object consists of known building blocks, the structural information could be further refined by the use of such knowledge. To this end, the particles were localized and replaced by 100 $\mathrm{nm}$ spheres to aid in the visualization of the morphology. Oversized spheres were represented by circles with dotted gray outlines. The right column in Figure 4 shows images of the localized spheres from a seven layer sample. The 
distance between layers in defect free areas matched well with the expected value of $\sqrt{2 / 3}$ times the diameter of the spheres. The projection image of layers taken three at a time are shown in the left-hand column. Starting with the first layer, spheres were colored in a repetitive sequence of red, green, and blue, respectively. Overlapping spheres in the projection image resulted in mixed colors. The net packing is r-hcp. Structural features such as grain boundaries and the organization around defects are plainly revealed.

In conclusion, we have demonstrated the direct 3D imaging of densely packed colloidal nanostructures using subdiffraction 3D resolution provided by STED microscopy. To our knowledge, no other technique currently affords the direct and local visualization of densely packed 3D structures on the nanoscale. By using an efficient combination of two deexcitation patterns, a simultaneous resolution of $43 \mathrm{~nm}$ in the lateral and $125 \mathrm{~nm}$ in the axial direction was achieved corresponding to an effective focal volume of $1.3 \mathrm{aL}$ that is 126 times smaller than that of a confocal microscope using the same wavelength. Imaging a model system of latex spheres organized by confined convective assembly revealed the unambiguous mapping of the resultant crystal structures, in particular the observation of fcc, hcp, r-hcp, and bcc structures. In the near future, we will extend our studies of colloids in this size regime to include the temporal evolution of crystal formation and the assembly of building blocks with more complex shapes and interaction forces where richer, more intriguing behavior is expected. Along with the data presented herein, these prospects highlight the enormous potential of subdiffraction far-field fluorescence microscopy for the sciences of colloids and 3D nanostructures.

Acknowledgment. This work was supported by the Alexander von Humboldt Foundation (C.K.U.). We thank Gael Moneron and Mariano Bossi for useful discussions and assistance. Additionally we thank A. Schönle for support with the IMSPECTOR software.

Note Added after ASAP Publication: This paper was published ASAP on January 1, 2008. Minor changes were made to the text. The revised paper was reposted on February $16,2008$.

Supporting Information Available: Details of the sample preparation, setup of the microscope, and modeling per- formed. This material is available free of charge via the Internet at http://pubs.acs.org.

\section{References}

(1) Hell, S. W. Science 2007, 316 (5828), 1153-1158.

(2) Westphal, V.; Hell, S. W. Phys. Rev. Lett. 2005, 94, 143903.

(3) Prasad, V.; Semwogerere, D.; Weeks, E. R. J. Phys.:Condens. Matter 2007, 19 (11).

(4) Van Blaaderen, A.; Wiltzius, P. Science 1995, 270 (5239), 1177-1179.

(5) Alsayed, A. M.; Islam, M. F.; Zhang, J.; Collings, P. J.; Yodh, A. G. Science 2005, 309 (5738), 1207-1210.

(6) de Villeneuve, V. W. A.; Dullens, R. P. A.; Aarts, D.; Groeneveld, E.; Scherff, J. H.; Kegel, W. K.; Lekkerkerker, H. N. W. Science 2005, 309 (5738), 1231-1233.

(7) Schall, P.; Cohen, I.; Weitz, D. A.; Spaepen, F. Nature 2006, 440 (7082), 319-323.

(8) Schall, P.; Cohen, I.; Weitz, D. A.; Spaepen, F. Science 2004, 305 (5692), 1944-1948.

(9) Glotzer, S. C.; Solomon, M. J. Nat. Mater. 2007, 6 (8), 557-562.

(10) van Blaaderen, A. Nature 2006, 439 (7076), 545-546.

(11) Velev, O. D. Science 2006, 312 (5772), 376-377.

(12) Shevchenko, E. V.; Talapin, D. V.; Kotov, N. A.; O’Brien, S.; Murray, C. B. Nature 2006, 439 (7072), 55-59.

(13) Leunissen, M. E.; Christova, C. G.; Hynninen, A. P.; Royall, C. P.; Campbell, A. I.; Imhof, A.; Dijkstra, M.; van Roij, R.; van Blaaderen, A. Nature 2005, 437 (7056), 235-240.

(14) Urban, J. J.; Talapin, D. V.; Shevchenko, E. V.; Kagan, C. R.; Murray, C. B. Nat. Mater. 2007, 6 (2), 115-121.

(15) Kalsin, A. M.; Fialkowski, M.; Paszewski, M.; Smoukov, S. K.; Bishop, K. J. M.; Grzybowski, B. A. Science 2006, 312 (5772), 420424.

(16) Hell, S. W. Nat. Biotechnol. 2003, 21 (11), 1347-1355.

(17) Hofmann, M.; Eggeling, C.; Jakobs, S.; Hell, S. W. Proc. Natl. Acad. Sci. U.S.A. 2005, 102 (49), 17565-17569.

(18) Hell, S. W.; Wichmann, J. Opt. Lett. 1994, 19 (11), 780-782.

(19) Keller, J.; Schönle, A.; Hell, S. W. Opt. Express 2007, 15 (6), 33613371.

(20) Donnert, G.; Keller, J.; Medda, R.; Andrei, M. A.; Rizzoli, S. O.; Lührmann, R.; Jahn, R.; Eggeling, C.; Hell, S. W. Proc. Natl. Acad. Sci. U.S.A. 2006, 103 (31), 11440-11445.

(21) Klar, T. A.; Jakobs, S.; Dyba, M.; Egner, A.; Hell, S. W. Proc. Natl. Acad. Sci. U.S.A. 2000, 97, 8206-8210.

(22) Denkov, N. D.; Velev, O. D.; Kralchevsky, P. A.; Ivanov, I. B.; Yoshimura, H.; Nagayama, K. Nature 1993, 361 (6407), 26-26.

(23) Dimitrov, A. S.; Nagayama, K. Langmuir 1996, 12 (5), 1303-1311.

(24) Jiang, P.; Bertone, J. F.; Hwang, K. S.; Colvin, V. L. Chem. Mater. 1999, 11 (8), 2132-2140.

(25) Norris, D. J.; Arlinghaus, E. G.; Meng, L. L.; Heiny, R.; Scriven, L. E. Adv. Mater. 2004, 16 (16), 1393-1399.

(26) Meng, L. L.; Wei, H.; Nagel, A.; Wiley, B. J.; Scriven, L. E.; Norris, D. J. Nano Lett. 2006, 6 (10), 2249-2253.

(27) Denkov, N. D.; Velev, O. D.; Kralchevsky, P. A.; Ivanov, I. B.; Yoshimura, H.; Nagayama, K. Langmuir 1992, 8 (12), 3183-3190.

(28) Kim, M. H.; Im, S. H.; Park, O. O. Adv. Funct. Mater. 2005, 15 (8), $1329-1335$.

(29) Prevo, B. G.; Velev, O. D. Langmuir 2004, 20 (6), 2099-2107.

NL073164N 